\title{
Efficiency Enhancement of PEDOT:PSS/Si Hybrid Solar Cells by Using Nanostructured Radial Junction and Antireflective Surface
}

\author{
Jheng-Yuan Chen, ${ }^{\dagger}$ Celal Con, ${ }^{\ddagger}$ Ming-Hung Yu, ${ }^{\dagger}$ Bo Cui, ${ }^{*}{ }^{\ddagger}$ and Kien Wen Sun ${ }^{\dagger} \dagger$ \\ ${ }^{\dagger}$ Department of Applied Chemistry, National Chiao Tung University, 1001 University Road, Hsinchu 30010, Taiwan \\ ${ }^{\ddagger}$ Department of Electrical and Computer Engineering and Waterloo Institute for Nanotechnology (WIN), University of Waterloo, \\ 200 University Avenue West, Waterloo, Ontario N2L 3G1, Canada
}

ABSTRACT: We demonstrate the implementation of a hybrid solar cell that comprises a surface nanostructured ntype $\mathrm{Si}$ and poly(3,4-ethylenedioxythiophene):poly(styrenesulfonate). The $\mathrm{Si}$ surface before deposition of the organic layer was nanostructured by using $\mathrm{CsCl}$ self-assembled nanoparticles as a hard mask and dry etching to form radial junction architectures and enhance light diffusion and absorption. Apart from the textured Si surface, processing parameters such as from metal-electrode shadow ratio, spincoating rate, and surfactant addition were properly adjusted to improve overall cell performance. Our hybrid solar cells achieve the best performance under optimized cell parameters with a power conversion efficiency of $8.84 \%$ and short-circuit current density of $30.5 \mathrm{~mA} / \mathrm{cm}^{2}$. This combined technique provides a simple, scalable, and cost-effective process for fabricating hybrid solar cells.

KEYWORDS: nanostructure, hybrid solar cell, cesium chloride, conductive polymer, light trapping, antireflective

\section{INTRODUCTION}

Crystalline Si has dominated photovoltaic markets for years because of its abundant material supply, nontoxicity, and high efficiency. However, the growth of the Si photovoltaic industry has been limited because of expensive fabrication processes and materials. Conventional Si solar cells have $p-n$ junctions for the efficient separation of light-generated charge carriers. The $p-n$ junction is normally formed by furnace diffusion, which is expensive and requires very high temperatures $\left(\sim 1000{ }^{\circ} \mathrm{C}\right)$. Organic solar cells that are created with conjugated polymers, which can be fabricated with inexpensive materials by using low temperature solution processes, and have the potential to lower costs; however, the efficiency of organic solar cells is lower than Si-based devices. Therefore, hybrid solar cells that combine Si and conjugated polymers at low temperatures provide a possible alternative technology to simplify fabrication processes and reduce costs. ${ }^{1-17}$

A conjugated polymer called poly (3, 4ethylenedioxythiophene):poly(styrenesulfonate) (PEDOT:PSS) is the most widely used organic material for hybrid devices. PEDOT:PSS is transparent, is conductive (1000 S/ $\mathrm{cm})$, and can produce a Schottky junction with $\mathrm{Si}^{12-17}$ Illuminative light is absorbed in n-type $\mathrm{Si}$, and a hole transport layer in PEDOT:PSS can extract holes generated in the Si substrate out of the device. Thus, the efficiency of the hybrid PEDOT:PSS/Si solar cell is comparable to a conventional Si p$\mathrm{n}$ junction solar cell in principle.

To reduce the cost of photovoltaic devices further, significant efforts have been made in reducing the thickness of the Si

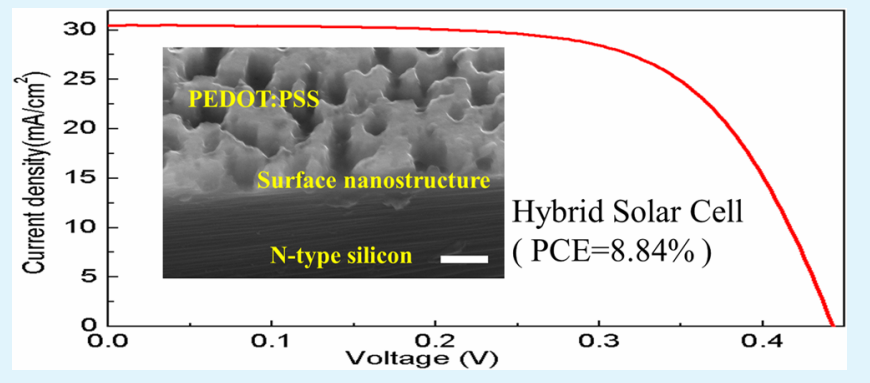

substrate, which requires considerable improvement in light absorption. The use of nanoscale surface structures which are more useful for thin-film devices than traditional microscale texturing for crystalline Si solar cells is a promising method to enhance light absorption. ${ }^{11,12}$ Nanoscale surface structures such as nanowire, ${ }^{10-12,14-17,20}$ nanorod, $^{21}$ and nanocone ${ }^{13,22}$ not only provide enhanced absorption properties by antireflective and light scattering effects but also offers a large junction area for charge separation and radial junction architecture. Radial junction-array solar cells allow efficient charge transport and high light-harvesting capabilities because of the decoupling of light absorption and charge-carrier collection directions. ${ }^{10-17,20-22}$ Nanoscale surface structures have been widely applied as antireflective coatings ${ }^{23,24}$ for crystalline $\mathrm{Si}$ solar cells, ${ }^{20,21}$ thin-film solar cells, ${ }^{22}$ organic solar cells, ${ }^{25}$ and hybrid solar cells ${ }^{10-17}$ because of their significant advantages.

Nanostructures can be fabricated by using various techniques that involve lithography and etching. Versatile techniques such as self-assembly lithography ${ }^{26-30}$ have recently emerged as promising candidates for fabricating nanostructures and reducing costs. Thin films consisting of self-assembled islands have been used as etching masks for the fabrication of surface nanostructures. These materials used in island films, which are typically metals, can be easily formed over large area. ${ }^{26-28}$ Compared with metals, cesium chloride $(\mathrm{CsCl})$ self-assembly

Received: May 16, 2013

Accepted: July 11, 2013

Published: July 11, 2013 


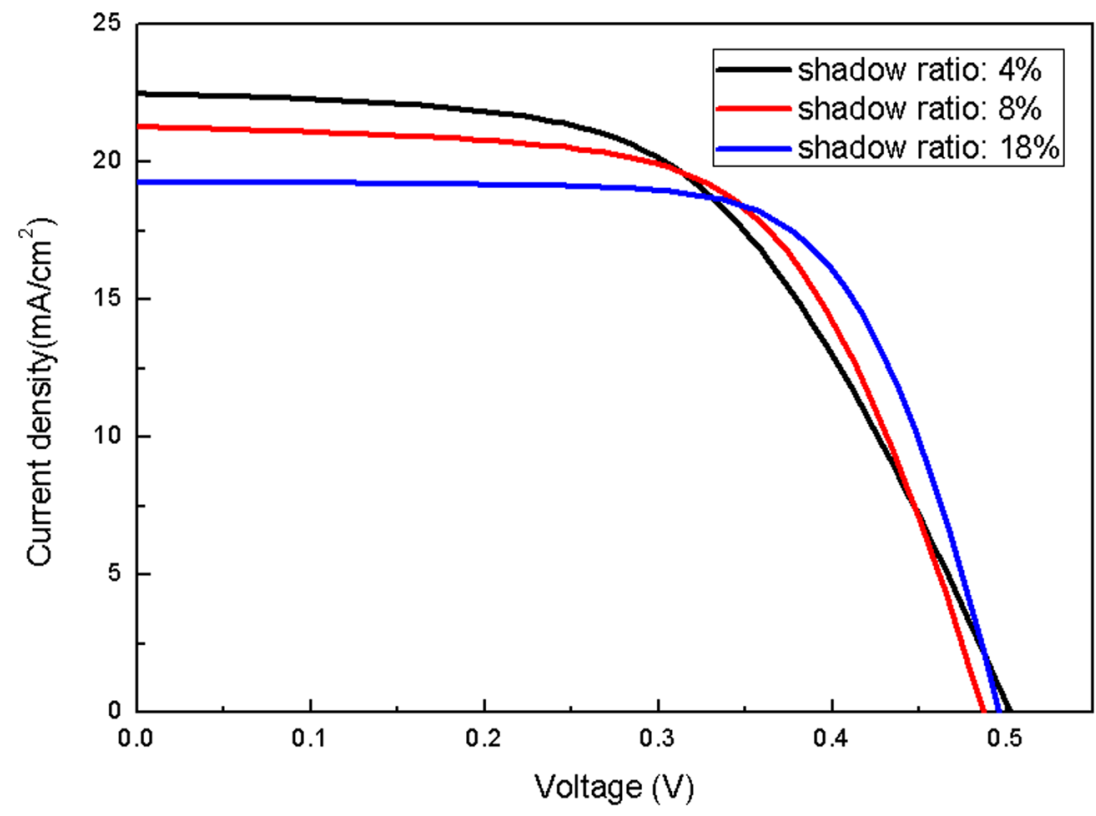

Figure 1. Current density-voltage $(J-V)$ characteristics of the devices with different shadow ratio of front metal electrode.

has been recently used to fabricate structures with larger distributions by controlling film thickness and relative humidity and by developing time. ${ }^{29,30} \mathrm{CsCl}$ can be easily removed with water; thus, this material does not leave behind any contaminants. However, one should note that the gaps between nanostructures should be controllable to allow polymers to infiltrate and form thin films on the Si surface. ${ }^{13}$

In this study, we demonstrate a hybrid solar cell that comprises a nanostructured n-type Si surface and PEDOT:PSS. The surface structure of $\mathrm{Si}$ was engineered by combining $\mathrm{CsCl}$ self-assembly and dry etching to form radial junction and antireflective architectures for light absorption enhancement.

\section{EXPERIMENTAL SECTION}

2.1. Fabrication of Si Nanostructure by Using $\mathrm{CsCl}$ SelfAssembly and Reactive Ion Etching (RIE). Nanostructures were fabricated on n-type, polished $\langle 100\rangle$ oriented $\mathrm{Si}$ wafers with $500 \mu \mathrm{m}$ thicknesses and $1 \Omega$-cm to $20 \Omega$-cm resistivity. First, a thin layer of $\mathrm{CsCl}$ was deposited by thermal evaporation. The wafers were then exposed to moisture to allow hemispherical $\mathrm{CsCl}$ islands to form by self-assembly. The size, shape, and density of the self-assembly can be adjusted by controlling the evaporated film thickness, exposure time of the film in air, and relative humidity of air. Two nanostructured samples were fabricated in this experiment, namely, NS1 and NS2, which had 20 and $10 \mathrm{~nm} \mathrm{CsCl}$ layer thickness, 50 and $10 \mathrm{~min}$ exposure times to air, and $12 \%$ and $40 \%$ relative humidity, respectively. Thereafter, these structures were transferred on $\mathrm{Si}$ by using $\mathrm{CF}_{4} \mathrm{RIE}$ for $2 \mathrm{~min}$ at $30 \mathrm{sccm}, 20 \mathrm{mT}$ orr, and $100 \mathrm{~W}$. Finally, the samples were washed by deionized water to remove residual $\mathrm{CsCl}$ and cleaned by oxygen plasma.

2.2. Hybrid Solar Cell Fabrication. The Si substrate after initial cleaning was dipped in dilute hydrofluoric (HF) acid (2\%) to remove native $\mathrm{SiO}_{2}$. Immediately after $\mathrm{HF}$ cleaning and drying, a $100 \mathrm{~nm}$ thick $\mathrm{Al}$ layer was deposited on the backside for cathode contact by using the sputter system. A highly conductive polymer solution was then prepared by mixing PEDOT:PSS (PH1000, Clevios) solution with 5 wt \% dimethyl sulfoxide (DMSO) as a secondary dopant to increase conductivity. PEDOT:PSS was deposited onto the Si substrates by spin coating followed by thermal annealing at $120{ }^{\circ} \mathrm{C}$ for $10 \mathrm{~min}$. Finally, the front anode contact was fabricated with a $60 \mathrm{~nm}$ thick $\mathrm{Ag}$ grid by electron beam evaporation through a steel foil shadow mask.

\section{RESULTS AND DISCUSSION}

To compare the performance of the hybrid solar cell with and without surface nanostructures, we first fabricated hybrid solar cells on a flat surface and optimized the efficiency with process parameters. The thickness of the organic PEDOT:PSS layer was controlled by the spin-coating rates. Under spin-coating rates of 1000, 3000, 5000, and $7000 \mathrm{rpm}$, the thicknesses of the PEDOT:PSS layer were 140, 70, 50, and $40 \mathrm{~nm}$, respectively. Although the PEDOT:PSS layer is sufficiently conductive for forming a Schottky junction with $\mathrm{Si}$ and transporting lightgenerated holes out of the Si substrate, the conductivity of the PEDOT:PSS layer is insufficient as a top electrode. Therefore, we employed a finger-grid thin film of $\mathrm{Ag}$ as the top anode. Figure 1 shows the current density-voltage $(J-V)$ characteristics of the devices with different front metal-electrode shadow ratios. The photocurrent of the solar cells was analyzed by using a solar simulator under air-mass 1.5 global (AM 1.5G) illumination conditions $\left(100 \mathrm{~mW} / \mathrm{cm}^{2}, 25{ }^{\circ} \mathrm{C}\right)$. The three tested devices have the same electrode-finger width of $100 \mu \mathrm{m}$. The thickness of the PEDOT:PSS layer was set to $70 \mathrm{~nm}$, which produced the best performance for all three devices. Devices with shadow ratios of $4 \%, 8 \%$, and $18 \%$ were controlled by using different finger periods of 2,1 , and $0.5 \mathrm{~mm}$, respectively. The short-circuit current density $\left(J_{\text {sc }}\right)$ decreases when the light absorption decreases with increasing shadow ratios. The fill factor (FF) improves from $54.9 \%$ (with a shadow ratio of $4 \%$ ) to $61.9 \%$ (with a shadow ratio of $8 \%$ ) and $69.1 \%$ (with a shadow ratio of $18 \%$ ). The improvement in FF is attributed to the decrease in series resistance from $5.2 \Omega \cdot \mathrm{cm}^{2}$ to $3.1 \Omega \cdot \mathrm{cm}^{2}$ and $2.4 \Omega \cdot \mathrm{cm}^{2}$ with increasing shadow ratios from $4 \%$ to $18 \%$. The device with the highest top electrode density shows the best power conversion efficiency (PCE) of $6.59 \%$ because of improvements in carrier collection even though the $J_{\text {sc }}$ decreases slightly. Therefore, all samples were fabricated by using top electrodes with shadow ratios of $18 \%$ throughout the experiment.

Nanostructures on the surface of the n-type Si substrate were fabricated by using $\mathrm{CsCl}$ self-assembly and dry etching. The $\mathrm{Si}$ substrate was then etched by RIE dry etching by using the $\mathrm{CsCl}$ 

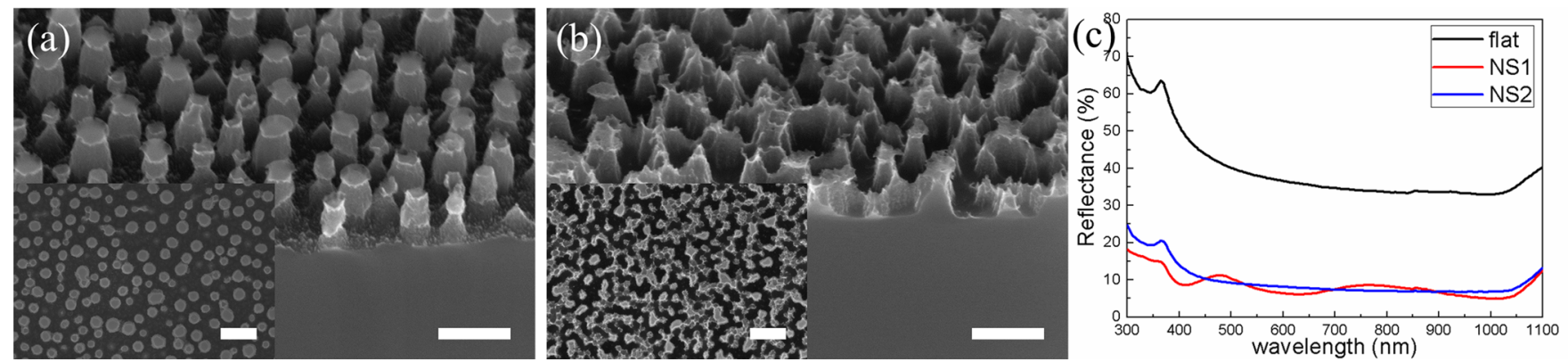

Figure 2. $45^{\circ}$ tilted side-view SEM images of (a) NS1 and (b) NS2. The inset is the top view of the structure. All scale bars are $500 \mathrm{~nm}$. (c) Reflectance spectra of different nanostructured profiles. The flat surface is also displayed in parallel for comparison.

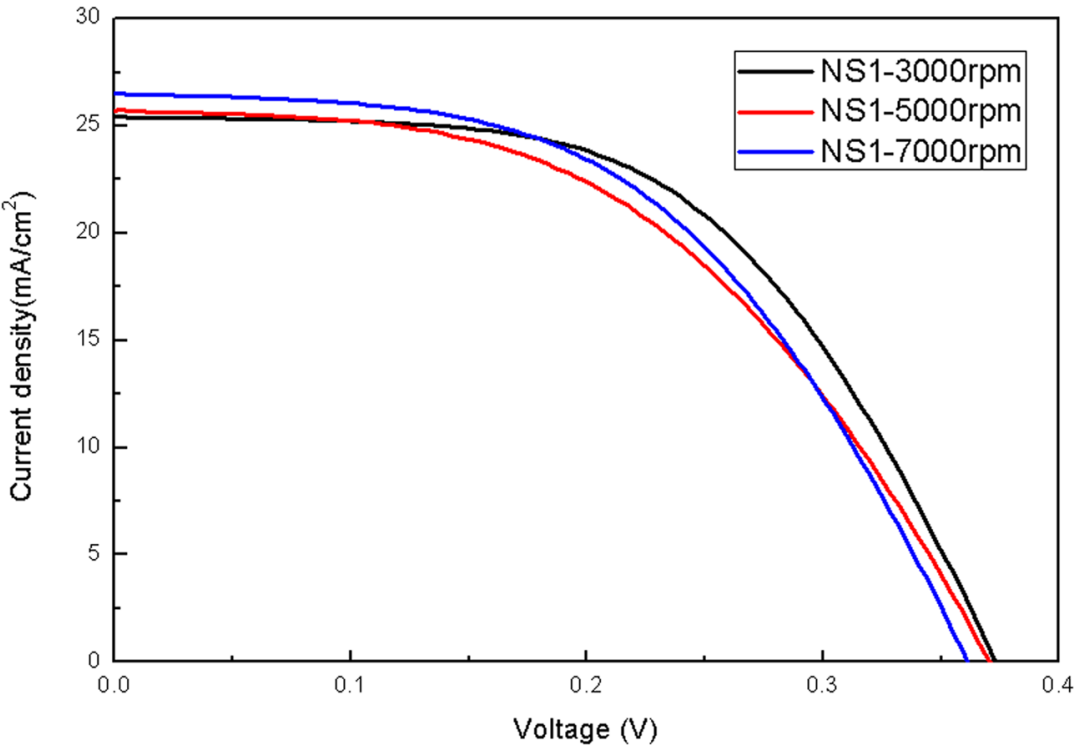

Figure 3. $J-V$ characteristics of the devices with different spin-coating rates on NS1 surface structure.

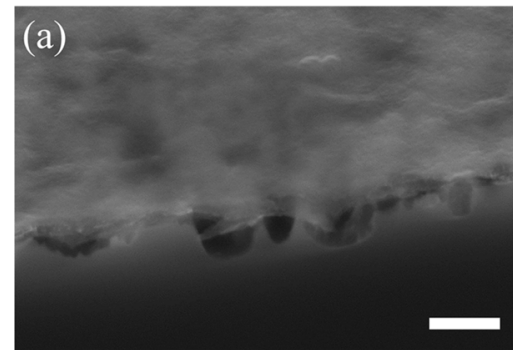

(b)

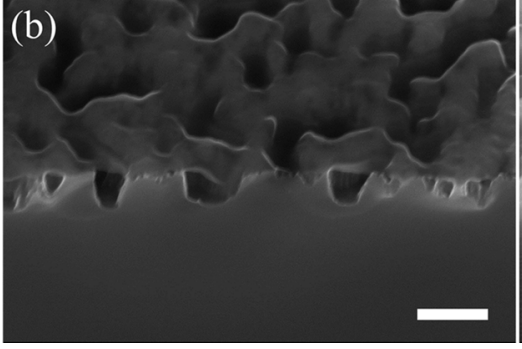

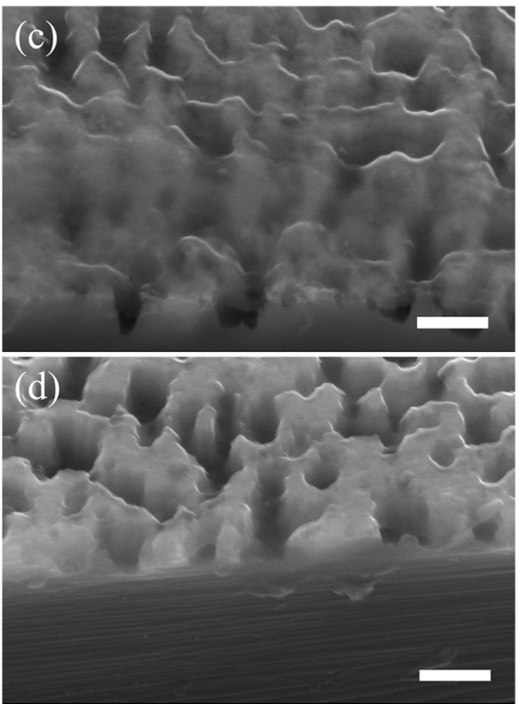

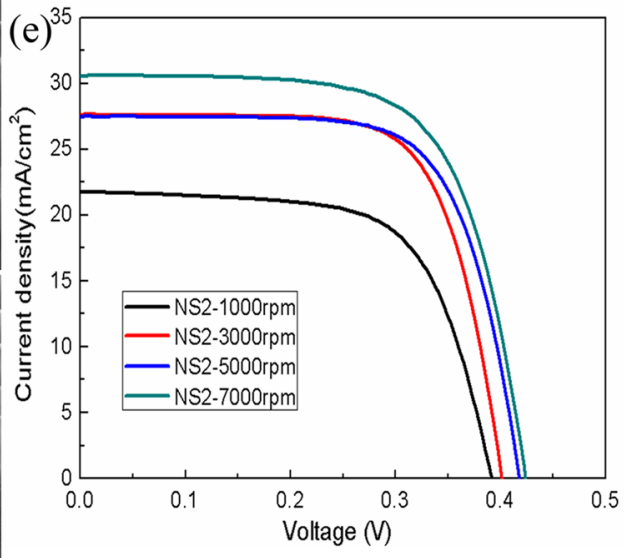

Figure 4. (a-d) $45^{\circ}$ tilted side-view SEM images of different spin-coating speeds: (a) $1000 \mathrm{rpm}$, (b) $3000 \mathrm{rpm}$, (c) $5000 \mathrm{rpm}$, and (d) $7000 \mathrm{rpm}$. All scale bars are $500 \mathrm{~nm}$. (e) $J-V$ characteristics of the devices with different spin-coating rates on NS2 surface structure.

islands as a hard mask. To achieve high light absorption and conformal polymer spin coating, the nanostructures on the Si surface should be smaller than the wavelength of incident light. $^{18,19}$ The formation of a tapered nanostructure profile is preferred. ${ }^{13,25}$ The pillar structures, which is referred as the NS1 structure in this paper, has a 100 to $200 \mathrm{~nm}$ diameter and $100 \mathrm{~nm}$ depth (scanning electron microscopy (SEM) image, Figure 2a). The reflectance spectra of the Si substrates with 

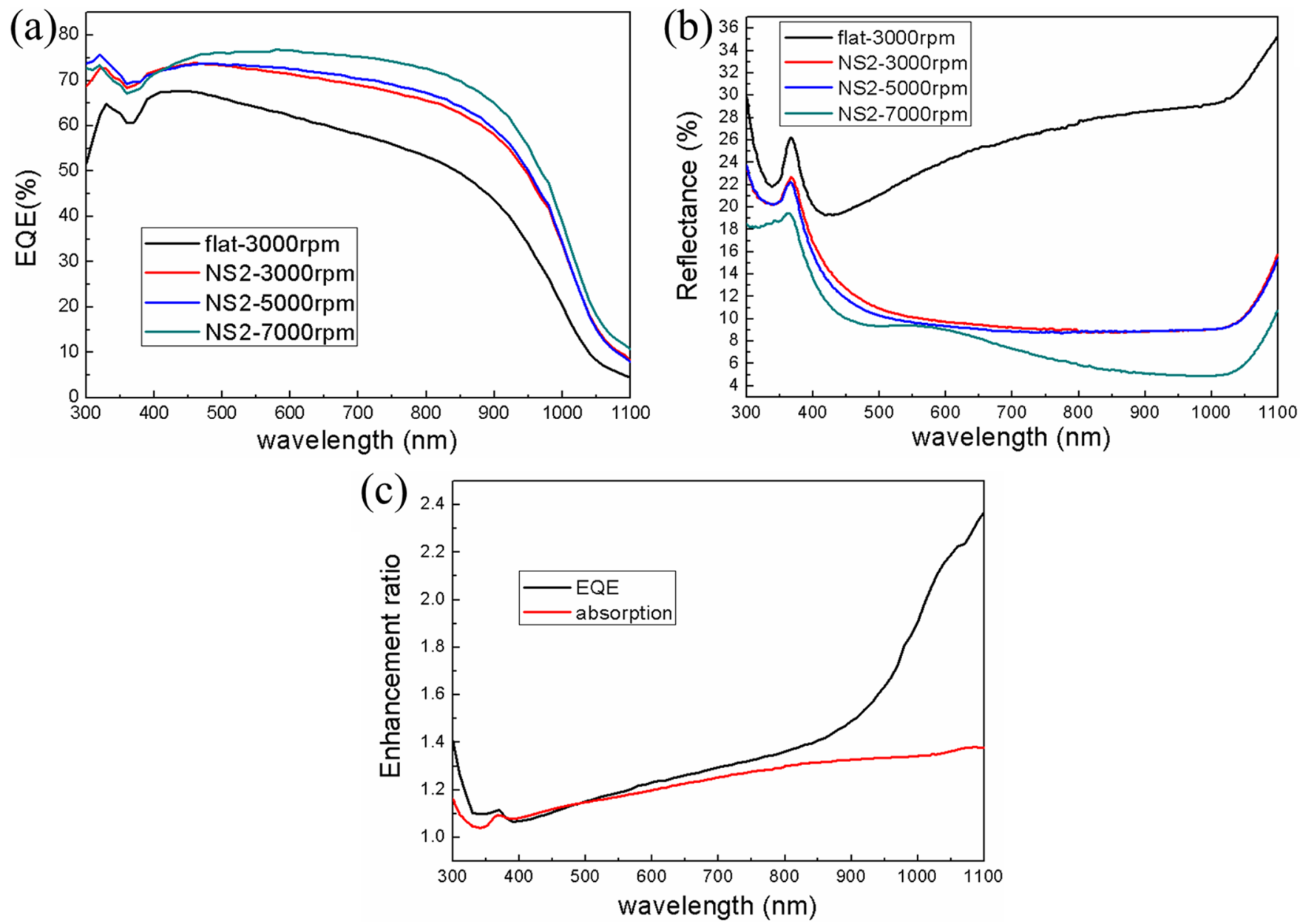

Figure 5. (a) EQE and (b) reflectance spectra of the hybrid solar cells fabricated with 3000, 5000, and 7000 rpm on NS2 surface structure, and the flat surface device with $3000 \mathrm{rpm}$ is also displayed in parallel for comparison. (c) Enhancement ratios in EQE and light absorption between the flat device and device made at a spin-coating rate of $7000 \mathrm{rpm}$.

nanostructured surfaces were measured by using a UV/visible/ NIR spectrophotometer with an integrating sphere for 300 to $1100 \mathrm{~nm}$ wavelengths. Figure 2c compares the reflectance spectra of samples with nanostructured profiles and flatsurfaced Si substrates. The flat-surfaced Si substrate shows a reflection above $30 \%$ throughout the entire spectra. The NS1 nanostructure reduces the reflectance by approximately $25 \%$ to $40 \%$; the reflectance is lower than $10 \%$ for wavelengths longer than $450 \mathrm{~nm}$. The average reflectance of the NS1 structure at a wavelength range of 300 to $1100 \mathrm{~nm}$ is $8.5 \%$, which is lower than the flat-surfaced Si substrate (39.5\%). The NS1 structure provides antireflective and light trapping effects to reduce the surface reflection and increase the absorption of the $\mathrm{Si}$ substrate.

Figure 3 shows the $J-V$ characteristics of the devices with different spin-coating rates of PEDOT:PSS in the NS1 structure. $J_{s c}$ was enhanced to above $25 \mathrm{~mA} / \mathrm{cm}^{2}$ because of increased light absorption compared with samples without nanostructures. However, the open-circuit voltage $\left(V_{\mathrm{oc}}\right)$ and FF of these devices decrease compared with flat-surfaced devices. The decrease of $V_{\mathrm{oc}}$ is attributed to the increasing interface area and void between the polymer and $\mathrm{Si}$, thus leading to increased surface recombination. ${ }^{13,14,17}$ The lower FF is caused by the nonuniform polymer coating with voids and surface defects (Figure 2a). The best PCE performance of devices with NS1 surface structures is only $5.21 \%$, which is lower than that of flat- surfaced samples. This result is caused by the deterioration of FF $(54.9 \%)$ and $V_{\text {oc }}(373 \mathrm{mV})$ even with increasing $J_{\text {sc }}(25.4$ $\left.\mathrm{mA} / \mathrm{cm}^{2}\right)$.

To improve the interface between the polymer and $\mathrm{Si}$, we further modified the surface structure of NS1. Figure $2 \mathrm{~b}$ shows the SEM image, which is referred to as the NS2 structure, with diameters from 100 to $150 \mathrm{~nm}$ and a depth of $100 \mathrm{~nm}$ in this study. By controlling the $\mathrm{CsCl}$ self-assembly process, the NS2 structure achieves a tapered shape and smooth walls, which are preferred for conformal polymer-surface coverage via spin coating. Surface reflection was also investigated to compare the NS2 structure and flat-surfaced Si substrate (Figure 2c). The reflectance of the NS2 structure is lower than $10 \%$ for wavelengths longer than $450 \mathrm{~nm}$. The average reflectance of the NS2 structure in the wavelength range of 300 to $1100 \mathrm{~nm}$ is $9.6 \%$, which is close to that of the NS1 structure.

We used spin coating with different speeds to deposit the polymer and adjust the PEDOT:PSS/Si interface. Figure $4 \mathrm{a}-\mathrm{d}$ shows the SEM images of the PEDOT:PSS morphology coated on the surface of the NS2 structure at speeds of 1000, 3000, 5000 , and $7000 \mathrm{rpm}$. Under a low spin-coating rate, the polymer formed a flat thin film with numerous voids between the polymer and Si (Figure 4a). The surface coverage of the polymer improves with increasing spin-coating rate. We were able to deposit the most conformal polymer film over the $\mathrm{Si}$ nanostructure under a spin-coating rate of $7000 \mathrm{rpm}$ (Figure 


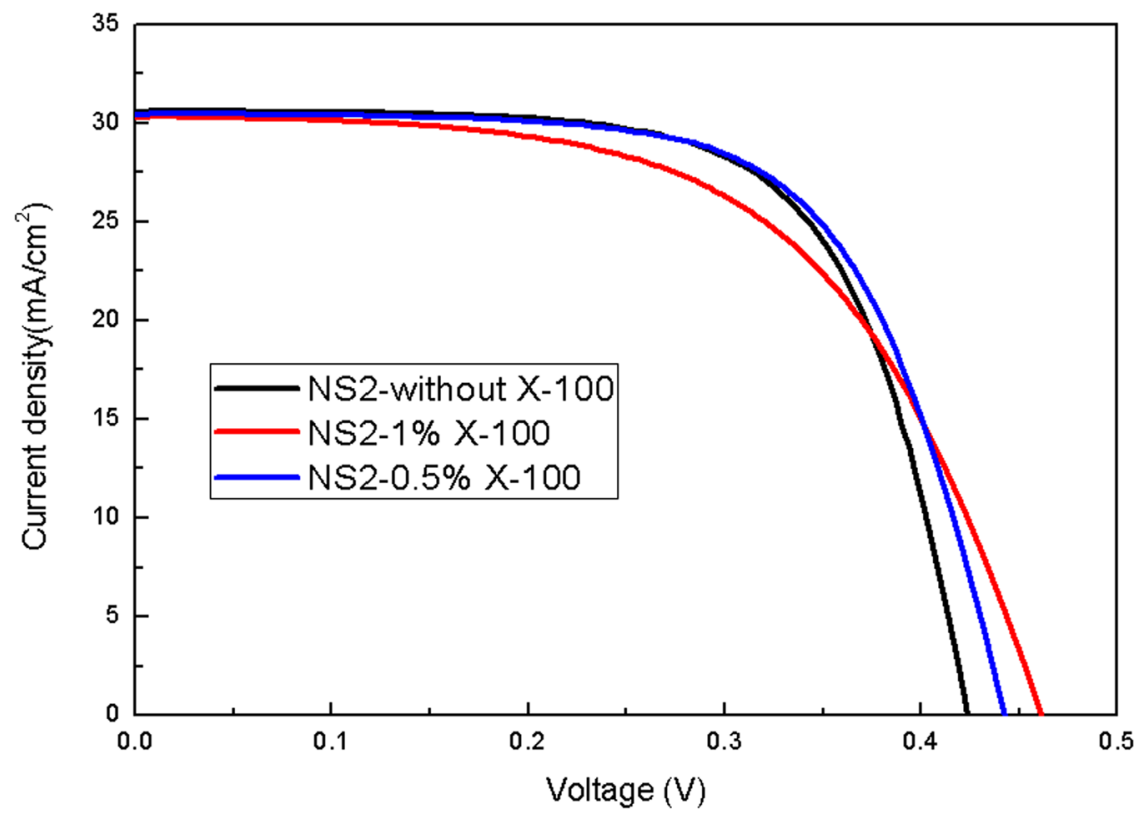

Figure 6. $J-V$ characteristics of the devices with and without Triton X-100 wetting agent.

$4 \mathrm{~d})$. Figure $4 \mathrm{e}$ shows the $J-V$ characteristics of the four devices with different spin-coating rates. Under the slowest speed, the device shows the lowest $V_{\mathrm{oc}}(391 \mathrm{mV})$ because the polymer is unable to fully infiltrate into the space between the $\mathrm{Si}$ nanostructures and form a continuous junction, thus leading to increased carrier recombination. ${ }^{13,14,17}$ Furthermore, the top surface is now smoother, thus reducing the antireflective effect. $J_{\text {sc }}$ reaches $21.8 \mathrm{~mA} / \mathrm{cm}^{2}$, which is a slight increase compared with that of flat-surface device. The PCE was only $5.60 \%$, which is lower than devices without surface nanostructures. The performance of solar cells gradually improves with increasing spin-coating rates. For devices at 3000 and $5000 \mathrm{rpm}, V_{\mathrm{oc}}$ increases to $401 \mathrm{mV}$ and $417 \mathrm{mV}$, respectively. $J_{\mathrm{sc}}$ increases above $27.5 \mathrm{~mA} / \mathrm{cm}^{2}$ because of the significant improvements in surface properties. The device fabricated at a spin-coating rate of $7000 \mathrm{rpm}$ shows the most conformal surface morphology and provides the best results with $V_{\text {oc }}$ at $424 \mathrm{mV}, J_{\text {sc }}$ at 30.6 $\mathrm{mA} / \mathrm{cm}^{2}$, and PCE at $8.70 \%$. The FFs of all four devices are within a close range (65\% to $70 \%)$, which is similar to devices without nanostructures. This result indicates that the parameters used for processing the NS2 structure are optimal for processing highly efficient hybrid solar cells.

Figure 5a shows the measured external quantum efficiency (EQE) spectra of the hybrid solar cells fabricated with spincoating rates of 3000,5000 , and $7000 \mathrm{rpm}$ by using the NS2 surface structure. A device built on a flat surface with a spincoating rate of $3000 \mathrm{rpm}$ is displayed in parallel for comparison. The flat-surfaced device shows a maximum EQE of $68 \%$ at 440 $\mathrm{nm}$. However, this EQE rapidly decreases with increasing wavelength because the longer wavelength light is absorbed far below the surface than that of the shorter wavelength light. Carriers generated deep in the substrate require a longer diffusion path in order to reach the junction for carrier separation. Therefore, this results in low separation efficiency and low EQE due to higher carrier recombination rates. Devices with nanostructured surfaces exhibit improved EQE performance throughout the entire wavelength range. This enhancement in EQE is even more significant at longer wavelengths and is attributed to the antireflective effect and the decoupling of light absorption and charge-carrier collection directions, thus allowing efficient charge transport and high light-harvesting capabilities. To make our argument more clear, the optical reflectance spectra of the nanostructured and flat surface $\mathrm{Si}$ after coating with the PEDOT:PSS are shown in Figure $5 \mathrm{~b}$. When comparing the EQE and reflectance spectra side by side, it is clear that antireflection effect indeed is the predominant reason for the $\mathrm{EQE}$ enhancement. The device made at the spin-coating rate of $7000 \mathrm{rpm}$ achieves the most conformal morphology, exhibiting an EQE above $70 \%$ over a wide wavelength range (i.e., between 400 and $850 \mathrm{~nm}$ ). This particular device also displays the lowest reflectance throughout the entire wavelength range (Figure 5b). In Figure 5c we show the enhancement ratios in EQE and light absorption between the flat device and device made at a spin-coating rate of 7000 $\mathrm{rpm}$. Note that the enhancement ratio in EQE is significantly higher than that of the light absorption at longer wavelengths. This indicates that the efficiency improvement is not only due to antireflective effect but also attributed to higher carrier collection efficiency at longer wavelengths. The enhanced carrier collection efficiency at long wavelength can be explained by the following reason. The scattering from the surface nanostructures increases the optical path length inside the silicon and also makes the light propagate laterally. This results to the enhanced absorption of long wavelength photons near the surface (light trapping effect) than that of the flat surface. ${ }^{13,21}$ Therefore, the device with nanostructures shows enhanced response at the longer wavelengths of the spectrum. This result indicates that the antireflective effect is no longer sensitive to the wavelength of incoming light. Surface nanostructures, along with the light trapping effect at long wavelengths, lead to the electron-hole pair generation near the surface that is easier to collect, thus resulting in high EQE.

Although our hybrid solar cells fabricated at $7000 \mathrm{rpm}$ on the NS2 surface structure show efficient PCE because of the high $J_{\text {sc }}$ the $V_{\text {oc }}$ values from the aforementioned devices are still lower than that of other reports. ${ }^{13-16}$ The decrease in $V_{\text {oc }}$ can be attributed to following reasons: (a) the low grade silicon wafers (with shorter carrier lifetimes) used in the experiments, 
(b) the increased interface area between the polymer and $\mathrm{Si}^{13}$ and (c) the air voids between the interfaces. (b) and (c) both lead to high surface recombination.

The Si surface becomes hydrophobic after HF cleaning, thus reducing the adhesion of polymer on $\mathrm{Si}$. We attempted to improve the adhesion of the polymer on $\mathrm{Si}$ by using a wetting agent (i.e., Triton X-100). ${ }^{16}$ Figure 6 shows the $J-V$ characteristics of the two devices that have additional spincoatings of $1 \%$ and $0.5 \%$ Triton X-100 at $7000 \mathrm{rpm}$ before PEDOT:PSS deposition. Compared with a device without a wetting agent, the $J_{s c}$ of the devices with wetting agents are almost unchanged; however, $V_{\mathrm{oc}}$ was slightly increased because the improvement of adhesion, and the FF was slightly reduced due to the nonconductive material involved, resulting in higher resistance. The PCE of the device treated with a $0.5 \%$ Triton X100 wetting agent further improves from $8.70 \%$ to $8.84 \%$.

We summarize the performances of devices made on NS2 structures and a flat surface in Table 1. Our best results show an

Table 1. Photovoltaic Properties of the Hybrid Si flat and NS2 Nanostructure Solar Cells

$\begin{array}{lccccc} & \begin{array}{c}V_{\text {oc }} \\ (\mathrm{mV})\end{array} & \begin{array}{c}J_{\text {sc }} \\ \left(\mathrm{mA} / \mathrm{cm}^{2}\right)\end{array} & \begin{array}{c}\mathrm{FF} \\ (\%)\end{array} & \begin{array}{c}\text { PCE } \\ (\%)\end{array} & \begin{array}{c}R_{\text {series }} \\ \left(\Omega \cdot \mathrm{cm}^{2}\right)\end{array} \\ \text { flat } & 496 & 19.3 & 69.1 & 6.59 & 2.36 \\ 1000 \mathrm{rpm} & 391 & 21.8 & 65.6 & 5.60 & 1.09 \\ 3000 \mathrm{rpm} & 401 & 27.6 & 70.3 & 7.78 & 0.65 \\ 5000 \mathrm{rpm} & 417 & 27.5 & 70.0 & 8.01 & 0.64 \\ 7000 \mathrm{rpm} & 424 & 30.6 & 67.1 & 8.70 & 0.42 \\ 1 \% \mathrm{X}-100 & 461 & 30.3 & 57.4 & 8.03 & 1.65 \\ 0.5 \% \text { X-100 } & 442 & 30.5 & 65.5 & 8.84 & 1.21\end{array}$

increase in $J_{s c}$ and the conversion efficiency by $58 \%$ and $34.1 \%$ (from $6.59 \%$ to $8.84 \%$ ) with optimized solar cell structures. We believe that improvements in $V_{\text {oc }}$ by using better metal contact and reducing surface recombination can advance the performance of polymer/silicon hybrid solar cells further.

\section{CONCLUSIONS}

We demonstrated the implementation of a PEDOT:PSS/Si solar cell that has a PCE of $8.84 \%$ and $J_{\mathrm{sc}}$ of $30.5 \mathrm{~mA} / \mathrm{cm}^{2}$. The nanostructures on the $\mathrm{Si}$ surface that was fabricated by using $\mathrm{CsCl}$ self-assembly and dry etching provide enhanced absorption and radial junction architecture. The front metalelectrode shadow ratio, spin-coating rate, and surfactant addition were also adjusted properly to achieve the best device performance. Nanostructures created by using $\mathrm{CsCl}$ selfassembly and dry-etching not only have the general advantages of self-assembly such as low cost and high throughput where an entire wafer can be patterned at a time but also possess unique superior qualities: wide tunable/controllable range of diameter and spacing by varying film thickness, relative humidity, and developing time. Furthermore, $\mathrm{CsCl}$ can be easily removed with water so that it does not leave behind any contaminants. Other than that, surface nanostructures cause high light absorption; thus, the device requires only a thin $\mathrm{Si}$ substrate, which can further reduce the cost of the solar cells. This combined technique provides a simple, scalable, and cost-effective process for the fabrication of efficient hybrid solar cells.

\section{AUTHOR INFORMATION}

\section{Corresponding Author}

*E-mail: bcui@uwaterloo.ca (Bo Cui); kwsun@mail.nctu.edu. tw (Kien Wen Sun).

\section{Author Contributions}

The manuscript was written through contributions of all authors. All authors have given approval to the final version of the manuscript.

\section{Notes}

The authors declare no competing financial interest.

\section{ACKNOWLEDGMENTS}

We thank Prof. Siva Sivoththaman and Dr. Bahareh Sadeghimakki of the Department of Electrical and Computer Engineering, University of Waterloo, Ontario, Canada, for their assistance on the reflection and EQE measurements. This work is supported by the National Science Council of the Republic of China (Contract nos. NSC 101-2917-I-009-005- and 101-2918I-009-002-) and the approaching Top University Program of the Ministry of Education of the Republic of China.

\section{REFERENCES}

(1) Sailor, M. J.; Ginsburg, E. J.; Gorman, C. B.; Kumar, A.; Grubbs, R. H.; Lewis, N. S. Science 1990, 249, 1146-1149.

(2) El-Nahass, M. M.; Zeyada, H. M.; Aziz, M. S.; Makhlouf, M. M. Thin Solid Films 2005, 492, 290-297.

(3) Williams, E. L.; Jabbour, G. E.; Wang, Q.; Shaheen, S. E.; Ginley, D. S.; Schiff, E. A. Appl. Phys. Lett. 2005, 87, 223504.

(4) Gowrishankar, V.; Scully, S. R.; McGehee, M. D.; Wang, Q.; Branz, H. M. Appl. Phys. Lett. 2006, 89, 252102.

(5) El-Nahass, M. M.; Zeyada, H. M.; Abd-El-Rahman, K. F.; Darwish, A. A. A. Sol. Energy Mater. Sol. Cells 2007, 91, 1120-1126.

(6) Wang, W.; Schiff, E. A. Appl. Phys. Lett. 2007, 91, 133504.

(7) Lin, C. H.; Tseng, S. C.; Liu, Y. K.; Tai, Y.; Chattopadhyay, S.; Lin, C. F.; Lee, J. H.; Hwang, J. S.; Hsu, Y. Y.; Chen, L. C.; Chen, W. C.; Chen, K. H. Appl. Phys. Lett. 2008, 92, 233302.

(8) Avasthi, S.; Lee, S.; Loo, Y.-L.; Sturm, J. C. Adv. Mater. 2011, 23, $5762-5766$

(9) Campbell, I. H.; Crone, B. K. J. Appl. Phys. 2009, 106, 113704.

(10) Zhang, F.; Sun, B.; Song, T.; Zhu, X.; Lee, S. Chem. Mater. 2011, 23, 2084-2090.

(11) Shen, X.; Sun, B.; Liu, D.; Lee, S.-T. J. Am. Chem. Soc. 2011, 133, 19408-19415.

(12) He, L.; Jiang, C.; Wang, H.; Lai, D.; Rusli. ACS Appl. Mater. Interfaces 2012, 4, 1704-1708.

(13) Jeong, S.; Garnett, E. C.; Wang, S.; Yu, Z.; Fan, Shanhui; Brongersma, M. L.; McGehee, M. D.; Cui, Y. Nano Lett. 2012, 12, 2971-2976.

(14) Syu, H.-J.; Shiu, S.-C.; Lin, C.-F. Sol. Energy Mater. Sol. Cells 2012, 98, 267-272.

(15) Zhang, F.; Song, T.; Sun, B. Nanotechnology 2012, 23, 194006.

(16) He, L.; Rusli; Jiang, C.; Wang, H.; Lai, D. IEEE Electron. Device Lett. 2011, 32, 1406-1408.

(17) Moiz, S. A.; Nahhas, A. M.; Um, H.-D.; Jee, S.-W.; Cho, H. K.; Kim, S.-W.; Lee, J.-H. Nanotechnology 2012, 23, 145401.

(18) Chattopadhyay, S.; Huang, Y. F.; Jen, Y. J.; Ganguly, A.; Chen, K. H.; Chen, L. C. Mater. Sci. Eng. R 2010, 69, 1-35.

(19) Li, Y.; Zhang, J.; Yang, B. Nano Today 2010, 5, 117-127.

(20) Garnett, E.; Yang, P. Nano Lett. 2010, 10, 1082-1087.

(21) Lu, Y.; Lal, A. Nano Lett. 2010, 10, 4651-4656.

(22) Ferry, V. E.; Verschuuren, M. A.; Lare, M. C. v.; Schropp, R. E. I.; Atwater, H. A.; Polman, A. Nano Lett. 2011, 11, 4239-4245.

(23) Chen, J. Y.; Chang, W.-L.; Huang, C. K.; Sun, K. W. Opt. Express 2011, 19, 14411-14419.

(24) Huang, C. K.; Sun, K. W.; Chang, W.-L. Opt. Express 2012, 20, A85-A93. 
(25) Pandey, A. K.; Aljada, M.; Velusamy, M.; Burn, P. L.; Meredith, P. Adv. Mater. 2012, 24, 1055-1061.

(26) Wang, S.; Yu, X. Z.; Fan, H. T. Appl. Phys. Lett. 2007, 91, 061105.

(27) Lin, G.-R.; Chang, Y.-C.; Liu, E.-S.; Kuo, H.-C.; Lin, H.-S. Appl. Phys. Lett. 2007, 90, 181923.

(28) Leem, J. W.; Chung, K. S.; Yu, J. S. Curr. Appl. Phys. 2012, 12, 291-298.

(29) Liao, Y.-X.; Yi, F.-T. Nanotechnology 2010, 21, 465302.

(30) Liu, J.; Ashmkhan, M.; Wang, B.; Yi, F. Appl. Surf. Sci. 2012, 258, $8825-8830$. 